\title{
Tofacitinib as a Therapeutic Option in the Treatment of Refractory Scleritis: A Case Report
}

\author{
Markus DV ${ }^{1}$, Canelas Beer $\mathrm{SM}^{2}$ and Moca Trevisani VF ${ }^{x_{3}}$ \\ ${ }^{1}$ Discipline of Internal Medicine, University Santo Amaro (UNISA), São Paulo, Brazil \\ ${ }^{2}$ Department of Ophthalmology, Federal University of São Paulo (UNIFESP), São Paulo, Brazil \\ ${ }^{3}$ Discipline of Rheumatology at the University Santo Amaro (UNISA), Adjunct Professor of the discipline of Urgency and Evidence- \\ Based Health at the Federal University of São Paulo (UNIFESP), São Paulo Brazil
}

*Corresponding author: Moca Trevisani VF, Full Professor of the Discipline of Rheumatology at the University Santo Amaro (UNISA), Adjunct Professor of the discipline of Urgency and Evidence-Based Health at the Federal University of São Paulo (UNIFESP); Rua Pedro de Toledo, 744 Vila Clementino, São Paulo, Brazil, Cep: 04039-002_Tel +55 11 987244244, E-mail: vmoca@ uol.com.br

Citation: Markus DV, Canelas Beer SM, Moca Trevisani VF (2021) Tofacitinib as a Therapeutic Option in the Treatment of Refractory Scleritis: A Case Report. J Case Rep Stud 9(1): 104. doi: 10.15744/2348-9820.9.104

Received Date: May 01, 2021 Accepted Date: June 22, 2021 Published Date: June 24, 2021

\section{Summary}

This was a 50-year-old patient with recurrent bilateral nodular anterior scleritis refractory to several treatments, including immunosuppressants and anti Tnfa. She was assessed for the different causes of immune-mediated and infectious diseases, but nothing was found. Tofacitinib was started $5 \mathrm{mg}$ twice a day, which led to the spacing of crises until their total disappearance. At present, the patient has been crisis-free for nine months. In conclusion, the use of Janus Kinase inhibitor (Tofacitinib) alone was effective in the treatment of refractory recurrent nodular anterior scleritis.

Keywords: Scleritis; JAK inhibitor; Tofacitinib 


\section{Introduction}

Scleritis is rather considered uncommon or relatively rare, with incidence rate of approximately 4 per 100,000; and prevalence of $5 / 100,000$ population. More frequent in women between the fifth and sixth decades [1]. There is an association with a systemic disease in 40-50\% of cases; infectious causes account for less than $10 \%$ of cases, and eye surgeries, neoplasms and medications are even rarer causes [2]. The differentiation between the inflammatory or infectious cause is very important to determine the treatment strategy. When a cause is not detected, it is deemed idiopathic [2-4]. Scleritis can be anterior or posterior, and the anterior type can be diffuse, nodular or necrotizing. They cause great discomfort and can lead to loss of visual acuity due to complications (perforation, glaucoma, cataract) [2-4]. In general, they are initially treated according to the cause of the disease with local and systemic anti-inflammatory drugs (steroidal and non-steroidal), intraocular injections of corticosteroids, and when there is no response, the use of immunosuppressants is indicated. In refractory cases, immunobiological agents, such as tumor necrosis factor inhibitors and anti-CD 20 are indicated [5-7]. Tofacitinib is a selective inhibitor of the Janus Kinase (JAKs) family capable of inhibiting JAK1 and JAK3. The Janus kinases play an important role in the intracellular signaling of several important cytokines in the pathogenesis of autoimmune diseases [8]. Their therapeutic role is well established in the treatment of immune-mediated inflammatory diseases, such as rheumatoid arthritis, psoriatic arthritis and ulcerative colitis [9-11]. The objective is to report the case of a patient with refractory scleritis using tofacitinib.

\section{Case Report}

A 50-year-old female patient presented in October 2015 with bilateral ocular hyperaemia, accompanied by palpebral swelling, pain, feeling of sand in the eyes, photophobia and severe headache. At first examination, the patient presented with tenderness and pain, which worsened with eye movements and radiated to the cheek. External examination demonstrated immobile, scleral nodule and intense hyperemia in addition a local violaceous hue. During slit-lamp examination, a temporal nodule was observed, with marked dilation of deep episcleral plexus, which was displaced above a scleral edema. Visual acuity was 20/20 in both eyes. She was diagnosed with bilateral anterior, non-necrotizing, non-infectious, nodular scleritis and treatment was started with corticosteroid eye drops with improvement. After being asymptomatic for 3 months, the patient had a patient had a relapse, again treated with corticosteroid eye drops and oral non-steroidal anti-inflammatory drugs for a short period with improvement. However, the episodes became recurrent, always following the same clinical pattern of presentation and already showing no response to the previously proposed treatment. She underwent an intense investigation carried out by the rheumatologist for immune-mediated and infectious systemic diseases. Full blood count, urea, creatinine, transaminases, protein electrophoresis, thyroid function tests, gluten and lactose intolerance test (research was carried out for the presence of Rheumatoid Factor, antinuclear factor, extractable nuclear antigen antibodies (ENA), anti-neutrophil cytoplasmic antibody, antiphospholipid, lupus, tuberculosis skin test, serological tests for Lyme disease, HIV, toxoplasmosis, syphilis, cytomegalovirus, hepatitis $C$ and $B$, angiotensin-converting enzyme were negative. Urinalysis was negative. Imaging: Chest X-ray, salivary gland ultrasound and spirometry were negative. The patient did not have any other clinical manifestations besides eye inflammation and did not use any medication. In March 2016, after new activity, the patient started, in addition to topical treatment, systemic corticosteroids at high doses $(1 \mathrm{mg} / \mathrm{kg} / \mathrm{day}$; total dose of $60 \mathrm{mg} /$ day) with a withdrawal schedule. Response to treatment was attained, but in an attempt to remove the corticosteroid, new crises occurred, at increasingly shorter intervals. In April 2018, immunosuppressive treatment with azathioprine 100mg/day was started, without adequate response and the dose was increased to $150 \mathrm{mg}$ and maintained for six months but again there was also no response. The patient continued to use oral corticosteroids and corticosteroid drops daily for four months, due to the frequency of the crises, and as adverse events the patient gained weight and developed hypertension due to corticosteroid use. Immunosuppression was increased by combining methotrexate $(20 \mathrm{mg} / \mathrm{d})$ to azathioprine $(150 \mathrm{mg} / \mathrm{d})$ for seven months), but the response was also unsatisfactory. Then treatment with Adalimumab (40 mg subcutaneous (SC) Every 2 weeks) and methotrexate (20mg/d) was started and maintained for ten months, but the crises continued and, in an attempt to improve the condition, therapy with intraocular injections with triamcinolone was performed, with no success. Treatment with Tofacitinib $5 \mathrm{mg}$ was initiated twice a day and the crises became sparse and of light intensity and she has currently been crisis-free for twelve months, without any side effects (Figures 1,2,3 and 4). 


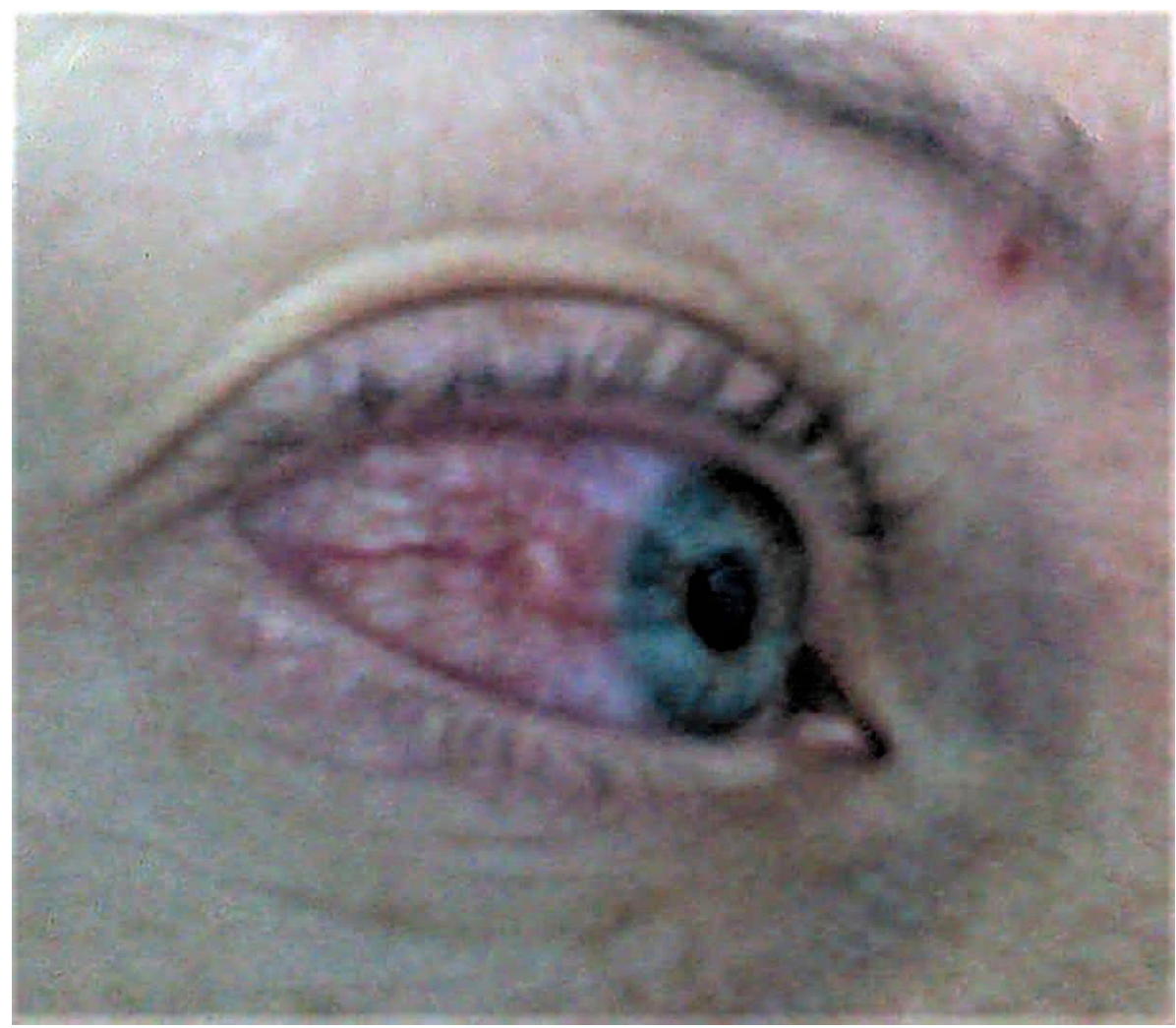

Figure 1: Nodular scleritis of right eye prior to tofacitinib therapy

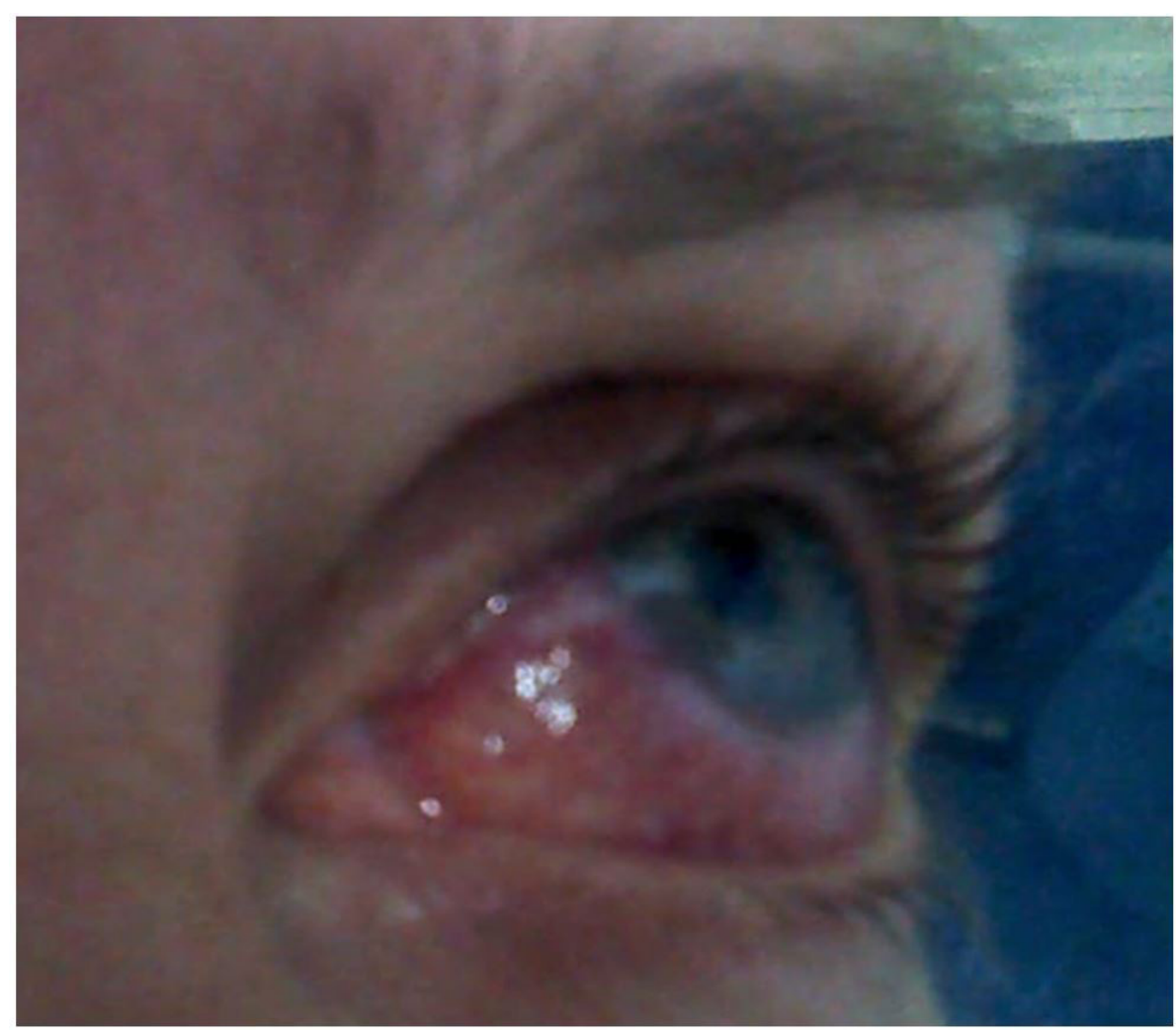

Figure 2: Scleritis of left eye prior to tofacitinib therapy 


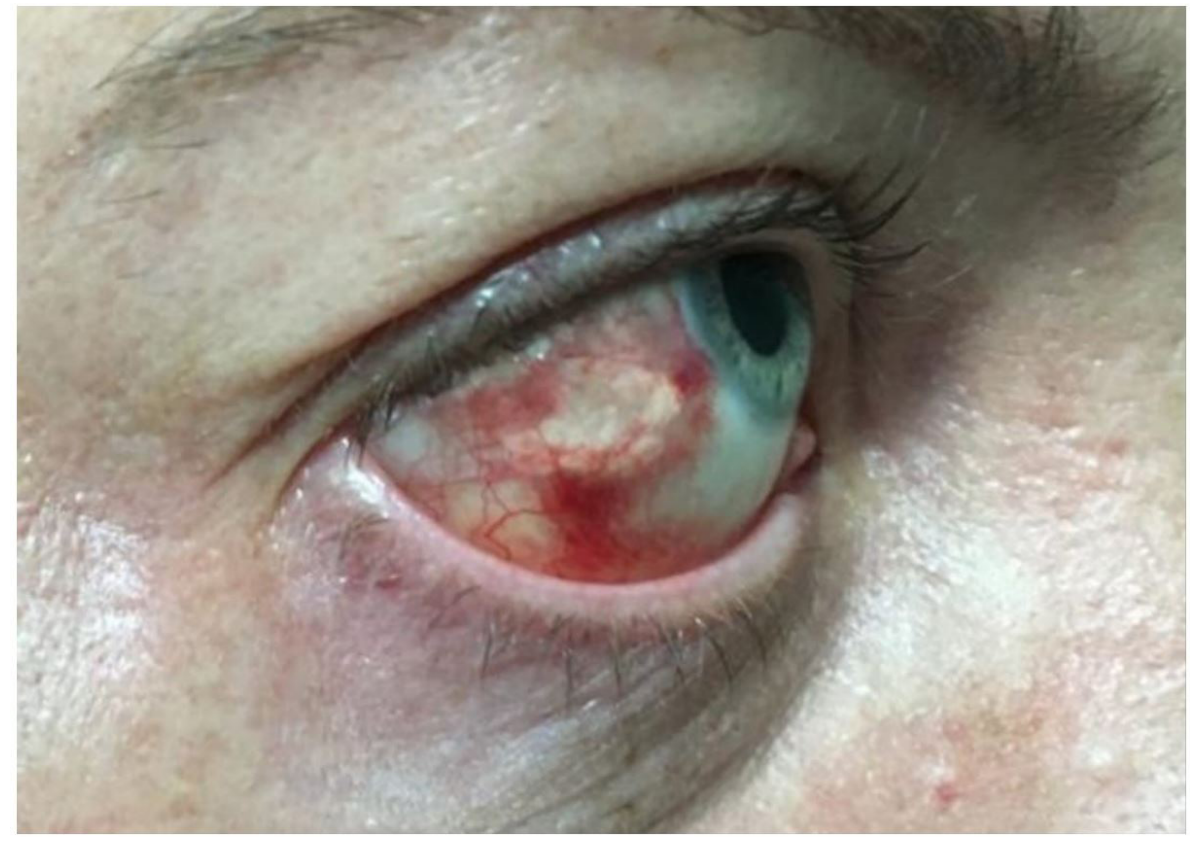

Figure 3: Right eye after the intraocular injection of triamcinolone

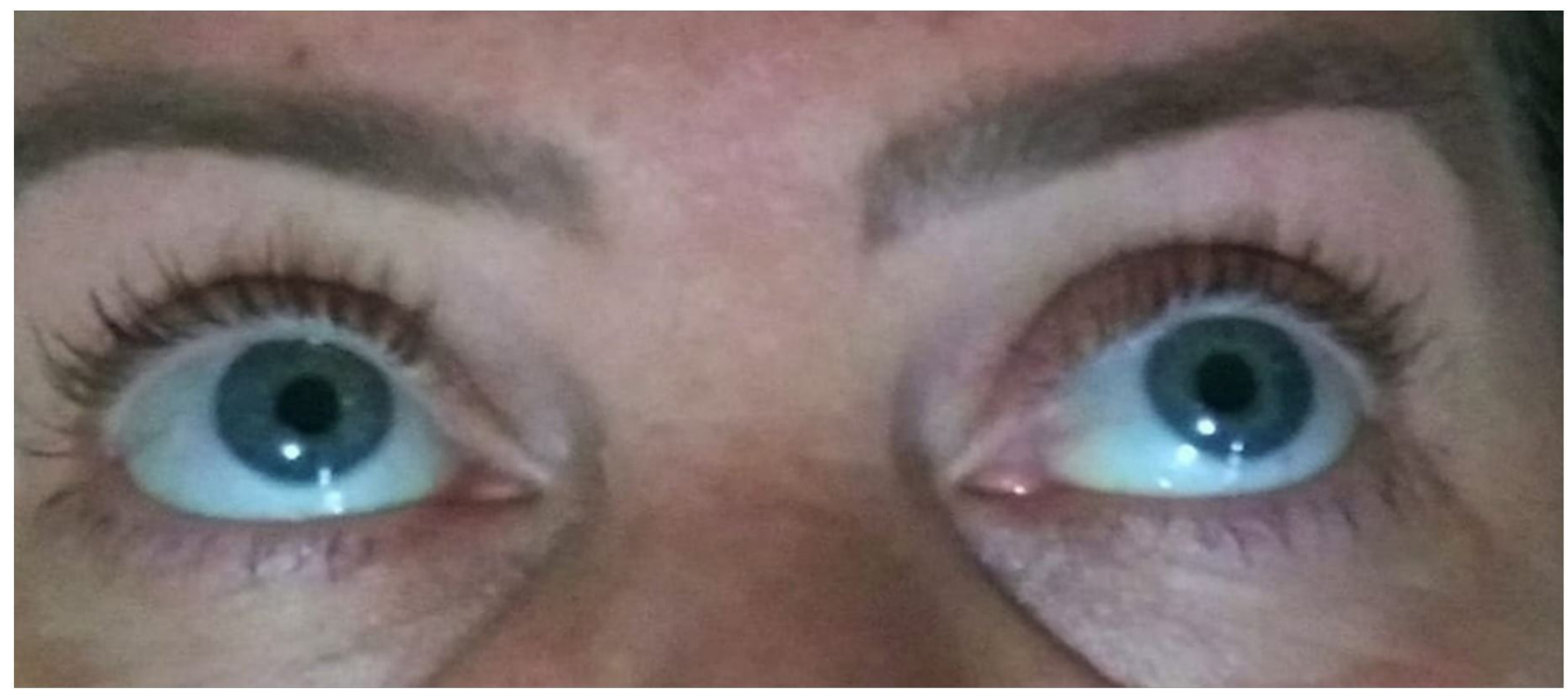

Figure 4: Image taken 12 months post tofacitinib therapy with full resolution of scleritis

\section{Discussion}

We report a case of a patient with recurrent bilateral idiopathic scleritis refractory to different treatments and who had an effective response to treatment with Tofacitinib. Recurrent and refractory scleritis is a severe inflammatory eye disease that causes high morbidity due to its possible complications. It is a challenge in clinical practice due to its prevalence, different forms of presentation and the fact that there are few randomized studies to guide treatment. Overall, the best level of evidence for treatment in these cases is based on reports or case series1-10. Immunohistochemistry studies on scleral tissue of patients with idiopathic scleritis have shown that most of the identified cells are macrophages, T cells (CD3 +, CD8 +), and B cells (CD20), and the role of cytokines and metalloproteinases has been demonstrated in the inflammatory process and in the destruction of scleral tissue [12]. Tofacitinib, by inhibiting Janus kinase 1 and 3, inhibits important inflammatory process pathways mediated by various cytokines and can play an important role in ocular inflammation [8]. Paley et al, reported the treatment of two patients with ocular inflammation, one 
with scleritis and the other with anterior and intermediate uveitis, who had refractory disease and showed an effective response to tofacitinib (in the case of the patient with scleritis, he needed the combination of tofacitinib and methotrexate) [13]. Pyare et al, reported a case of a 65-year-old woman with uveitis and necrotizing scleritis, refractory to other drugs, who achieved good control of the condition with the use of tofacitinib associated with prednisone and mycophenolate mofetil [14]. The third report in the literature is a 22-year-old patient diagnosed with juvenile idiopathic arthritis with anterior uveitis complicated by bilateral macular edema refractory to conventional treatment and who showed an effective response to tofacitinib [15]. In agreement with the rarely reported cases, this report demonstrates that the use of Tofacitinib as a therapy for refractory scleritis was effective by progressively decreasing the number of crises and their severity, in addition to reducing the need for corticosteroid use. It is necessary to conduct a randomized study with an appropriate number of patients to confirm the observed results.

\section{Conclusion}

Scleritis is a potentially severe disease that can lead to several eye complications and is often refractory to conventional therapy. In this report, after the failure of several treatments, Tofacitinib was shown to be an effective alternative therapy for crisis control and as a steroid sparing agent. 


\section{References}

1. E 1. Honik G, Wong IG, Gritz DC (2013) Incidence and prevalence of episcleritis and scleritis in Northern California. Cornea 32: $1562-6$.

2. Cunningham ET Jr, McCluskey P, Pavesio C, Wakefield D, Zierhut M (2016) Scleritis. Ocul Immunol Inflamm 24: 2-5.

3. Wakefield D, Di Girolamo N, Thurau S (2013) Scleritis: immunopathogenesis and molecular basis for therapy. Prog Retin Eye Res 35: 44-62.

4. Bielefeld P, Saadoun D, Héron E, Abad S, Devilliers H, et al. (2018) Scleritis and systemic diseases: What should know the internist? [Sclérites et maladies systémiques : que doit savoir l’interniste ?]. Rev Med Interne 39: 711-20.

5. Daniel Diaz J, Sobol EK, Gritz DC (2016) Treatment and management of scleral disorders. Surv Ophthalmol 61: 702-17.

6. Sands DS, Chan SCY, Gottlieb CC (2018) Methotrexate for the treatment of noninfectious scleritis. Can J Ophthalmol 53: 34953.

7. de Fidelix TS, Vieira LA, de Freitas D, Trevisani VF (2015) Biologic therapy for refractory scleritis: a new treatment perspective. Int Ophthalmol 35: 903-12.

8. Xu P, Shen P, Yu B, Xu X, Ge R, et al. (2020) Janus kinases (JAKs): The efficient therapeutic targets for autoimmune diseases and myeloproliferative disorders. Eur J Med Chem 192: 112155.

9. van der Heijde, Désirée (2019) “Tofacitinib in Combination With Methotrexate in Patients With Rheumatoid Arthritis: Clinical Efficacy, Radiographic, and Safety Outcomes From a Twenty-Four-Month, Phase III Study." Arthritis \& Rheumatology (Hoboken, N.J.) 71: 878-891.

10. Gladman D, Rigby W, Azevedo VF, Behrens F, Blanco R, et al. (2017) Tofacitinib for Psoriatic Arthritis in Patients with an Inadequate Response to TNF Inhibitors. N Engl J Med 377: 1525-36.

11. Panés J, Vermeire S, Lindsay JO (2019) Tofacitinib in Patients with Ulcerative Colitis: Health-Related Quality of Life in Phase 3 Randomised Controlled Induction and Maintenance Studies [published correction appears in J Crohns Colitis. 2019 Jan 1;13(1):139-140]. J Crohns Colitis 12: 145-56.

12. Wakefield D, Di Girolamo N, Thurau S, Wildner G, McCluskey P (2013) Scleritis: Immunopathogenesis and molecular basis for therapy. Prog Retin Eye Res 35: 44-62.

13. Paley MA, Karacal H, Rao PK, Margolis TP, Miner JJ (2018) Tofacitinib for refractory uveitis and scleritis. Am J Ophthalmol Case Rep 13: 53-5.

14. Pyare R, Kaushik V, Dutta Majumder P, Biswas J (2020) Tofacitinib in recalcitrant scleritis: First case report from India. Indian J Ophthalmol 68: 1988-90.

15. Heiligenhaus A, Heinz C (2019) Effect of Janus Kinase Inhibitor Treatment on Anterior Uveitis and Associated Macular Edema in an Adult Patient with Juvenile Idiopathic Arthritis. Ocul Immunol Inflamm 27: 1232-4. 


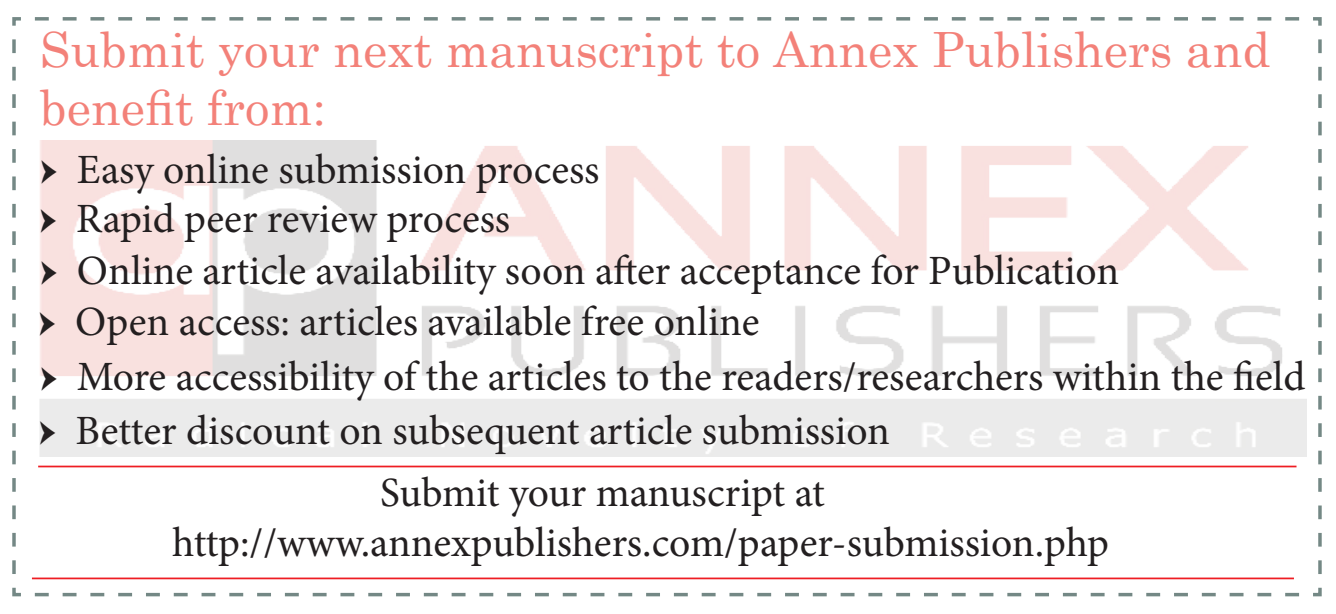

